\title{
WEIGHTED ESTIMATES FOR MARCINKIEWICZ INTEGRALS WITH NON-SMOOTH KERNELS ON SPACES OF HOMOGENEOUS TYPE AND THEIR APPLICATIONS
}

\author{
HUNG VIET LE
}

\begin{abstract}
Given a family of operators that act like approximations of the identity, we obtain weighted estimates for the Marcinkiewicz integrals with non-smooth kernels on spaces of homogeneous type. As applications of weighted estimates, we also establish the boundedness of these operators on the homogeneous Herz spaces over Euclidean spaces, and on the homogeneous weak Herz spaces for the endpoint case. We also study the boundedness of commutators of Marcinkiewicz integrals and BMO functions on various spaces.
\end{abstract}

Mathematics subject classification (2010): 42B20, 42B25, 42B35.

Keywords and phrases: Commutators, Muckenhoupt weights, Marcinkiewicz integrals, singular integrals, sharp maximal functions, BMO functions, Herz spaces, spaces of homogeneous type.

\section{REFERENCES}

[1] R. R. Coifman And G. Weiss, Extensions of Hardy spaces and their use in analysis, Bull. Amer. Math. Soc. 83 (1977), pp. 569-645.

[2] R. R. Coifman et G. Weiss, Analyse harmonique non-commutative sur certains espaces homogènes, Lecture Notes in Math. 242, Springer, Berlin (1971).

[3] X. T. DuONG AND A. McInTosh, Singular integral operators with non-smooth kernel on irregular domains, Revista Matemática Iberoamericana, 15, no. 2 (1999), pp. 233-265.

[4] X. T. DuOng AND L. YAn, Commutators of BMO functions and singular integral operators with non-smooth kernels, Bull. Austral. Math. Soc. 67 (2003), pp. 187-200.

[5] X. T. Duong And D. Robinson, Semigroup kernels, Poisson bounds and holomorphic functional calculus, J. Funct. Anal. 142 (1996), pp. 89-128.

[6] L. Grafakos, Classical and Modern Fourier Analysis, Prentice Hall, Upper Saddle River N. J., (2004).

[7] L. GRAFAKOS, L. LiU AND D. YANG, Vector-valued singular integrals and maximal functions on spaces of homogeneous type, Mathematica Scandinavica 104, no. 2 (2009), pp. 296-310.

[8] C. HERZ, Lipschitz spaces and Bernstein's theorem on absolutely convergent Fourier transforms, J. Math. Mech. 18 (1967), pp. 283-324.

[9] G. HU, S. LU AND D. YANG, Boundedness of rough singular integrals operators on homogeneous Herz spaces, J. Aust. Math. Soc. (Series A) 66 (1999), pp. 201-223.

[10] G. Hu, S. Lu AND D. YANG, The weak Herz spaces, J. Beijing Normal Univ. (Natur. Sci.) 33 (1997), pp. 27-34.

[11] G. HU AND D. YANG, Weighted estimates for singular integral operators with non-smooth kernels and applications, J. Aust. Math. Soc. 85 (2008), pp. 377-417.

[12] H. V. LE, Marcinkiewicz integrals with non-smooth kernels on spaces of homogeneous type, Analysis Mathematica 41 (2015), pp. 17-43.

[13] H. V. LE, Vector-Valued Singular Integrals with Non-smooth Kernels on Spaces of Homogeneous Type, Complex Analysis and Operator Theory 11, no. 1 (2016), pp. 57-84.

[14] H. V. LE, Commutators of Lipschitz. Functions and Singular Integrals with Non-Smooth Kernels on Euclidean Spaces, Analysis in Theory and Applications 32, no. 2 (2016), pp. 135-148. 
[15] Hui-Xia Mo and Shan-Zhen Lu, Vector-valued Singular Integral Operators with Non-smooth Kernels and Related Multilinear Commutators, Pure and Applied Mathematics Quarterly 3, no. 2 (2007), pp. 451-480.

[16] J. M. MARTELL, Sharp maximal functions associated with approximations of the identity in spaces of homogeneous type and applications, Studia Mathematica 161 (2004), pp. 113-145.

[17] J. GARCÍA-CUERVA AND J. L. RUBio DE FRANCIA, Weighted norm inequalities and related topics, North-Holland (1985).

[18] J.-O. Strömberg And A. Torchins Ky, Weighted Hardy Spaces, Lecture Notes in Mathematics 1381, Springer-Verlag, (1989).

[19] JING-SHI XU, Multilinear commutators of singular integral operators with non-smooth kernels, Taiwanese Journal of Mathematics 11, no. 2 (2007), pp. 483-496. 